\title{
A Note on Turán Numbers for Even Wheels
}

\section{Tomasz Dzido}

Received: 16 October 2011 / Revised: 18 July 2012 / Published online: 8 August 2012 (C) The Author(s) 2012. This article is published with open access at Springerlink.com

\begin{abstract}
The Turán number $\operatorname{ex}(n, G)$ is the maximum number of edges in any $n$-vertex graph that does not contain a subgraph isomorphic to $G$. We consider a very special case of the Simonovits's theorem (Simonovits in: Theory of graphs, Academic Press, New York, 1968) which determine an asymptotic result for Turán numbers for graphs with some properties. In the paper we present a more precise result for even wheels. We provide the exact value for Turán number ex $\left(n, W_{2 k}\right)$ for $n \geq 6 k-10$ and $k \geq 3$. In addition, we show that $\operatorname{ex}\left(n, W_{6}\right)=\left\lfloor\frac{n^{2}}{3}\right\rfloor$ for all $n \geq 6$. These numbers can be useful to calculate some Ramsey numbers.
\end{abstract}

Keywords Turán problem · Cycles

Mathematics Subject Classification (2000) $05 C 35 \cdot 05 C 38$

\section{Introduction}

In this paper, all graphs considered are undirected, finite and contain neither loops nor multiple edges. Let $G$ be such a graph. The vertex set of $G$ is denoted by $V(G)$, the edge set of $G$ by $E(G)$, and the number of edges in $G$ by $e(G)$. Let $d(v)$ be the degree of vertex $v, \Delta(G)$ be the maximum degree of vertices of $G$ and $\chi(G)$ be the chromatic number of graph $G$. $C_{m}$ denotes the cycle of length $m$. A wheel $W_{n}$ is a graph on $n$ vertices obtained from a $C_{n-1}$ by adding one vertex $w$ and making $w$ adjacent to all vertices of the $C_{n-1}$. Next notation follow that of Simonovits [4]. Let us consider the following graph: $n$ vertices are divided into $d$ classes each of which contains almost

T. Dzido $(\varangle)$

Institute of Informatics, University of Gdańsk, Wita Stwosza 57, 80-952 Gdańsk,

Poland

e-mail: tdz@inf.ug.edu.pl 
the same number of vertices: they contain $\left\lfloor\frac{n}{d}\right\rfloor$ or $\left\lfloor\frac{n}{d}+1\right\rfloor$ vertices. Join two vertices by an edge if and only if they belong to different classes. The graph obtained thus is denoted by $T^{n, d}$.

The Turán number ex $(n, G)$ is the maximum number of edges in any $n$-vertex graph that does not contain a subgraph isomorphic to $G$. A graph on $n$ vertices is said to be extremal with respect to $G$ if it does not contain a subgraph isomorphic to $G$ and has exactly ex $(n, G)$ edges.

The aim of this paper is to determine the Turán numbers of wheels $W_{n}$ for even $n$.

\section{Known Results}

First, we recall a result, which was proved by Mantel in 1907.

Theorem 1 (Mantel, [3]) The maximum number of edges in an n-vertex triangle-free graph is $\left\lfloor\frac{n^{2}}{4}\right\rfloor$

By Theorem 1 and since $W_{3}=C_{3}$, it is easy to have the property that for all integers $n, n \geq 3, \operatorname{ex}\left(n, W_{3}\right)=\left\lfloor\frac{n^{2}}{4}\right\rfloor$.

The famous Turán's theorem may be stated as follows.

Theorem 2 (Turán, [5]) Let $G$ be any subgraph of $K_{n}$ such that $G$ is $K_{r+1}$-free. Then the number of edges in $G$ is $e(G)=e\left(T^{n, r}\right)=\left\lfloor\frac{(r-1) n^{2}}{2 r}\right\rfloor$. In particular, $\operatorname{ex}\left(n, K_{4}\right)=\left\lfloor\frac{n^{2}}{3}\right\rfloor$.

As a special case, for $r=2$, one obtains Mantel's theorem. Since $W_{4}=K_{4}$, we obtain that for all integers $n, n \geq 3, \operatorname{ex}\left(n, W_{4}\right)=\left\lfloor\frac{n^{2}}{3}\right\rfloor$.

The remaining part of this paper is connected with the result of M. Simonovits.

Theorem 3 (Simonovits, [4]) Let $G$ be a given graph such that $\chi(G) \geq d+1$ but there is an edge e in it such that $\chi(G-\{e\})=d(G$ is color critical). Then there exists an $n_{0}$ such that if $n>n_{0}$ then $T^{n, d}$ is the only extremal graph with respect to $G$.

One can observe that the wheel $W_{2 k}$ is color critical, so it has Turán number equal to the Turán number of $K_{4}$ for sufficiently large $n$. In this paper we determine how large $n$ is.

The following theorems and corollaries will be used to prove the main results of this paper.

Theorem 4 (Woodall, [6]) Let $G$ be a graph on $n(n \geq 3)$ vertices with more than $\frac{n^{2}}{4}$ edges. Then $G$ contains a cycle of length $k$ for each $k\left(3 \leq k \leq\left\lfloor\frac{1}{2}(n+3)\right\rfloor\right)$.

Now, it is a simple observation that if graph $G$ has more than $\frac{n^{2}}{4}$ edges and $2 k-1 \leq$ $\left\lfloor\frac{1}{2}(n+3)\right\rfloor$, then $G$ contains a cycle of length $2 k-1$. Since $K_{\left\lfloor\frac{n}{2}\right\rfloor,\left\lceil\frac{n}{2}\right\rceil}$ contains no odd cycles, the maximum number of edges in a graph containing no $C_{2 k-1}$ (the Turán number for $C_{2 k-1}$ ) is $\left\lfloor\frac{n^{2}}{4}\right\rfloor$ for sufficiently large $n$. By these considerations we easily obtain the following 
Corollary 5 For all integers $k, n, n \geq 3,2 k-1 \leq\left\lfloor\frac{1}{2}(n+3)\right\rfloor$

$$
\operatorname{ex}\left(n, C_{2 k-1}\right)=\left\lfloor\frac{n^{2}}{4}\right\rfloor
$$

Moreover, another formula is known for $\operatorname{ex}\left(n, C_{2 k-1}\right)$. We give this, as the following theorem which can be found in the appendix IV of [1].

Theorem 6 ([1]) Assume that $2 k-1 \geq \frac{1}{2}(n+3)$. Then

$$
e x\left(n, C_{2 k-1}\right)=\left(\begin{array}{l}
n-(2 k-1)+2 \\
2
\end{array}\right)+\left(\begin{array}{l}
(2 k-1)-1 \\
2
\end{array}\right)
$$

One can see that the above theorem holds for $n \leq 4 k-5$. Let us consider the situation where $n=4 k-6$. By using Theorem 6 we obtain the property that ex $\left(4 k-6, C_{2 k-1}\right)=$ $\left(\begin{array}{c}2 k-3 \\ 2\end{array}\right)+\left(\begin{array}{c}2 k-2 \\ 2\end{array}\right)=(2 k-3)^{2}=\frac{(4 k-6)^{2}}{4}=\frac{n^{2}}{4}$. Since Corollary 5 holds for $n \geq 4 k-5$, then one can observe that for all $n \geq 4 k-6$ we have the following

Theorem 7 For all integers $k, n, n \geq 3, n \geq 4 k-6$

$$
\operatorname{ex}\left(n, C_{2 k-1}\right)=\left\lfloor\frac{n^{2}}{4}\right\rfloor
$$

\section{Main Result}

Now, we prove the following main result

Theorem 8 For all $k \geq 3$ and $n \geq 6 k-10$,

$$
\operatorname{ex}\left(n, W_{2 k}\right)=\left\lfloor\frac{n^{2}}{3}\right\rfloor
$$

Proof Let $G$ be a graph which does not contain a $W_{2 k}$ and assume that $G$ has $n \geq$ $6 k-10$ vertices and $e(G)=\left\lfloor\frac{n^{2}}{3}\right\rfloor+1$ edges. One can observe that $\Delta(G) \geq n-\left\lfloor\frac{n}{3}\right\rfloor$ since $\frac{\left(n-\left\lfloor\frac{n}{3}\right\rfloor-1\right) n}{2}<\left\lfloor\frac{n^{2}}{3}\right\rfloor+1$.

Suppose that there is a vertex $v \in V(G)$ such that $d(v)=n-1$. Then $G-v$ has exactly $\left\lfloor\frac{n^{2}}{3}\right\rfloor+1-(n-1)$ edges. By Theorem 7 we know that for all $n \geq 4 k-6$ we have that $\operatorname{ex}\left(n, C_{2 k-1}\right)=\left\lfloor\frac{n^{2}}{4}\right\rfloor$ and for all $k \geq 3$ we have $6 k-11>4 k-6$. We immediately obtain a contradiction with $\operatorname{ex}\left(n-1, C_{2 k-1}\right)=\left\lfloor\frac{(n-1)^{2}}{4}\right\rfloor$ since $\left\lfloor\frac{n^{2}}{3}\right\rfloor+1-(n-1)>$ $\left\lfloor\frac{(n-1)^{2}}{4}\right\rfloor$ for all $n \geq 6$. In general case, let us consider the situation when $\Delta(G)=n-p$ where $1 \leq p \leq\left\lfloor\frac{n}{3}\right\rfloor$ and there exists a vertex $v$ of degree $n-p$ in graph $G$. By Theorem 7 we know that for all $n \geq 4 k-6$ we have that $\operatorname{ex}\left(n, C_{2 k-1}\right)=\left\lfloor\frac{n^{2}}{4}\right\rfloor$ and for all $n \geq 6 k-10$ we have that $\Delta(G) \geq n-\left\lfloor\frac{n}{3}\right\rfloor \geq 4 k-6$. Let us consider a subgraph $G^{\prime}$ with $n-p$ vertices obtained by removing a vertex $v$ and $p-1$ vertices not adjacent 
to $v$ from the set of vertices of $G$. In this situation, the minimum number of edges in subgraph $G^{\prime}$ is $e\left(G^{\prime}\right) \geq e(G)-p(n-p)=\left\lfloor\frac{n^{2}}{3}\right\rfloor+1-p(n-p)$. We prove that $\left\lfloor\frac{n^{2}}{3}\right\rfloor+1-p(n-p)>\left\lfloor\frac{(n-p)^{2}}{4}\right\rfloor=\operatorname{ex}\left(n-p, C_{2 k-1}\right)$. One can calculate that formula $p(n-p)+\left\lfloor\frac{(n-p)^{2}}{4}\right\rfloor$ achieves the maximum value for $p=\left\lfloor\frac{n}{3}\right\rfloor$. The maximum value of this formula is equal to $\left\lfloor\frac{n^{2}}{3}\right\rfloor$. We obtain that $p(n-p)+\left\lfloor\frac{(n-p)^{2}}{4}\right\rfloor \leq\left\lfloor\frac{n^{2}}{3}\right\rfloor$, so we have a contradiction with our assumption about the number of edges in graph $G$ and $\operatorname{ex}\left(n, W_{2 k}\right) \leq\left\lfloor\frac{n^{2}}{3}\right\rfloor$.

Now, we show a graph $G$ on $n$ vertices and $\left\lfloor\frac{n^{2}}{3}\right\rfloor$ edges which does not contain a $W_{2 k}$. Let graph $G$ be $T^{n, 3}$. One can observe that $G$ is tripartite graph and does not contain a wheel of order $2 k$. The number of edges of this graph was calculated above and it is equal to $\left\lfloor\frac{n^{2}}{3}\right\rfloor$, so $\operatorname{ex}\left(n, W_{2 k}\right) \geq\left\lfloor\frac{n^{2}}{3}\right\rfloor$ and the proof is complete.

All values of $\operatorname{ex}\left(n, C_{5}\right)$, where $5 \leq n \leq 23$, are determined in [7], so by Theorem 7 and [7] we obtain the following

Corollary 9 Let $n \geq 5$. Then

$$
\text { ex }\left(n, C_{5}\right)= \begin{cases}7 & \text { if } n=5 \\ \left\lfloor\frac{n^{2}}{4}\right\rfloor & \text { otherwise }\end{cases}
$$

Now, we prove the following result.

Theorem 10 For all $n \geq 6$,

$$
\operatorname{ex}\left(n, W_{6}\right)=\left\lfloor\frac{n^{2}}{3}\right\rfloor
$$

Proof Let $G$ be a graph of order 6. If $G$ has at least 13 edges, then it contains a vertex $v \in V(G)$ such that $d(v)=5$. $G-v$ has at least 8 edges and we immediately obtain a contradiction with $\operatorname{ex}\left(5, C_{5}\right)=7$. We have that $\operatorname{ex}\left(6, W_{6}\right)=12=\frac{6^{2}}{3}$.

Let us consider a graph $G$ on 7 vertices which have $\left\lfloor\frac{7^{2}}{3}\right\rfloor+1=17$ edges. It is easy to see that $\Delta(G) \geq 5$. Suppose that there is a vertex $v \in V(G)$ such that $d(v)=6$. Then $G-v$ has exactly 11 edges and we immediately obtain a contradiction with $\operatorname{ex}\left(6, C_{5}\right)=9$. Now, suppose that there is a vertex $v \in V(G)$ such that $d(v)=5$. There is a vertex $w$ which not adjacent to $v$. Since $\operatorname{ex}\left(5, C_{5}\right)=7$, if the number of edges in a subgraph of $G$ on 5 vertices obtained by removing the vertices $v$ and $w$ from $G$ is at least 8 , we have a $W_{6}$. Consider the last case when $d(v)=d(w)=5$. In [2] we find a characterization of the extremal graphs of order 5 without a $C_{5}$. There are two such graphs: $\left(K_{3} \cup K_{1}\right)+K_{1}$ and $K_{2}+\overline{K_{3}}$. These graphs contain a vertex of degree 6 , a contradiction. We obtain a $W_{6}$, so $\operatorname{ex}\left(7, W_{6}\right)=16=\left\lfloor\frac{7^{2}}{3}\right\rfloor$ and an extremal graph is a complete tripartite graph $K_{2,2,3}$.

By using Theorem 8 we obtain the remaining part of proof. 
Open Access This article is distributed under the terms of the Creative Commons Attribution License which permits any use, distribution, and reproduction in any medium, provided the original author(s) and the source are credited.

\section{References}

1. Bondy, A.J., Murty, S.U.: Graph Theory with Applications. American Elsevier Publishing Co., New York (1976)

2. Caccetta, L., Vijayan, K.: Maximal cycles in graphs. Discret. Math. 98, 1-7 (1991)

3. Mantel, W.: Problem 28, soln. by H. Gouventak, W. Mantel, J. Teixeira de Mattes, F. Schuh and W.A. Wythoff. Wiskundige Opgaven 10, 60-61 (1907)

4. Simonovits, M.: A method for solving extremal problems in graph theory, stability problems. In: Theory of Graphs. Academic Press, New York (1968)

5. Turán, P.: On an extremal problem in graph theory. Mat. Fiz. Lapok 48, 436-452 (1941)

6. Woodall, R.D.: Sufficient conditions for circuits in graphs. Proc. Lond. Math. Soc. 24, 739-755 (1972)

7. Yang, Y., Rowlinson, P.: On the third Ramsey numbers of graphs with five edges. J. Combin. Math. Combin. Comput. 11, 213-222 (1992)

8. Radziszowski, S.P.: Small Ramsey numbers. Electron. J. Combin., Dynamic Survey 1, revision \#13. http://www.combinatorics.org. Accessed August 2011 